\title{
Paclitaxel production by endophytic fungus, Neopestalotiopsis clavispora KY624416 and subsequent extraction of chitosan from fungal biomass wastes
}

\author{
Koutb M.M. ${ }^{1,2}$, Hassan E.A. ${ }^{1 *}$, El-Sokkary, G.H. ${ }^{3}$, Saber S.H. ${ }^{3}$ and Hussein N.A. ${ }^{1}$ \\ ${ }^{1}$ Department of Botany and Microbiology, Faculty of Science, Assiut University, 71516, Assiut, Egypt \\ 2Department of Biology, Faculty of Applied Science, Umm Al-Qura University, Saudi Arabia \\ ${ }^{3}$ Department of Zoology, Faculty of Science, Assiut University, 71516, Assiut, Egypt \\ Received: 26/06/2021, Accepted: 28/09/2021, Available online: 04/10/2021 \\ *to whom all correspondence should be addressed: e-mail: elhagaghassan@ymail.com; elgaghassan@aun.edu.eg \\ https://doi.org/10.30955/gnj.003839
}

\section{Graphical abstract}

Paclitaxel and chitosan production from the endophytic strain, Neopestalotiopsis clavispora ASU1

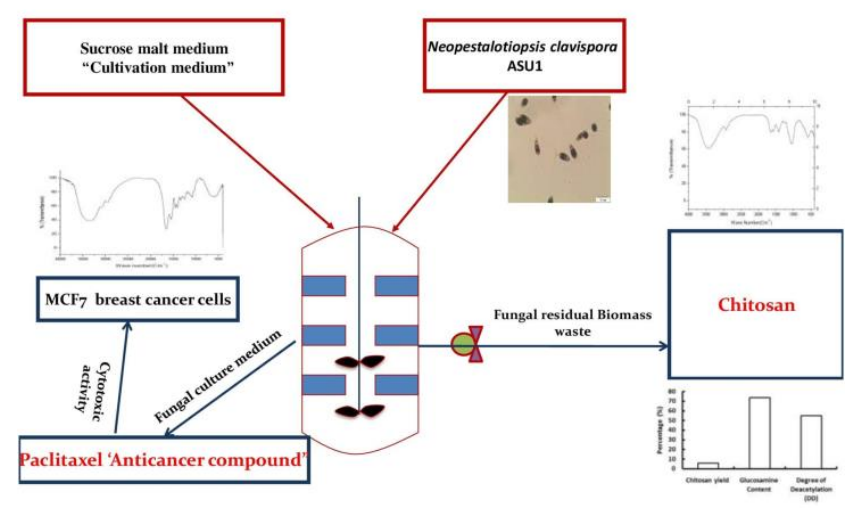

Abstract

Nowadays, breast cancer is considered to be one of the most prevalent disease worldwide, this initiated interest and growing demand for taxol production in large quantities. The limited availability of traditional taxol production from the Pacific yew trees has encouraged research into the development of taxol production from alternative sources. Thus, the current study aimed to investigate the chemopreventive effect of paclitaxel derived from endophytic fungus Neopestalotiopsis clavispora ASU1. The endophytic fungus Neopestalotiopsis clavispora ASU1(KY624416) showed potent productivity of paclitaxel recording $100.6 \mu \mathrm{g} / \mathrm{l}$ which was confirmed by HPLC, LC/Ms-Ms and FTIR analyses. In vitro, the extracted fungal taxol exerted a significant cytotoxic effect $(P<0.05)$ at $300 \mathrm{nM}$, revealed that the increase in paclitaxel concentration induced increasing in cell death. Furthermore, the present study provided a promising approach for coupling paclitaxel production technology by endophytic fungus $N$. clavispora with chitosan production from residual fungal biomass resulting from taxol extraction and therefore improve the feasibility and commercialization of taxol production. The chitosan yield represented $5.94 \%$ of residual fungal dry biomass. Also, fungal chitosan was characterized for the degree of deacetylation (DD) (54.60\%), FTIR spectroscopy, reducing power activity $(0.263 \pm 0.051 \mathrm{mg} / \mathrm{ml})$ may be attributed to hydroxyl groups $(\mathrm{OH})$, and amine groups. These results confirmed that fungi are promising alternative sources for chitosan with superior physiochemical characteristics for food and medical prospective applications.

Keywords: Anticancer, Chitosan, MCF7 cell line, Neopestalotiopsis clavispora, Paclitaxel.

\section{Introduction}

Paclitaxel is a natural diterpenic alkaloid compound and one of the most successful anticancer drugs (Brown, 2003; Suffness, 1995; Vasundhara et al., 2016). It is used for the treatment of some cancer diseases, such as ovarian, breast, leukemias, head and neck carcinoma, lymphomas and lung cancers as well as Kaposi's sarcoma (Brown, 2003; Chakravarthi et al., 2008; Croom Jr, 1995; Kharwar et al., 2011; Vennila et al., 2010). The mode of action of paclitaxel is to promote the assembly of microtubule-polymers and reduce the critical concentration of purified tubulin subunits necessary for polymerization into microtubules, leading to the failure of mitosis. Furthermore, it may alter other cellular functions, such as intracellular signaling, organelle transport and locomotion (Alexandre et al., 2007; Weaver, 2014). Paclitaxel was isolated from the bark of Taxus brevifolia, the Pacific yew tree (Heinig et al., 2013; Perdue, 1969; Wani et al., 1971). But, unfortunately, very low quantities of produced taxol and the destruction of trees are considered the major obstacles associated with paclitaxel production from the Pacific yew tree (Gallego et al., 2019). So, the produced amount of paclitaxel cannot meet the increasing demand for anticancer taxol compound on the market (Chakravarthi et al., 2008), leads to increase the efforts to develop alternative sources of taxol production, such as chemical synthesis, but this is very expensive and gives low yields (Frense, 2007; Guenard 
et al., 1993). This has encouraged researchers to explore other taxol candidates and develop technologies that generate a high yield of taxol from sustainable resources. Microorganisms are considered to be one of the most environmentally acceptable, relatively simple and costcompetitive paclitaxel feedstocks as they are characterized by a fast growth with high cell densities, easy scale-up, shear stress resistance, simple genetic manipulation, and dependable and unrestrained paclitaxel production. The last two aspects make microorganisms important requisites for industrial production and increase paclitaxel fermentation technology. Interestingly, over 30 taxolproducing fungi have been reported from previously published papers, the majority are the endophytes of Taxus spp. (Yuan et al., 2006). Thus, endophytic fungal fermentation processes may be an alternative way to produce taxol (Wang et al., 2000). With respect to Pestalotiopsis, several taxol-producing isolates were recovered from bald cypress in South Carolina (Strobel et al., 1997). Also, it was produced from Pestalotiopsis microspora, P. pausiceta, P. terminaliae and Chaetomella raphigera isolated from Taxus wallichiana, Taxodium distichum, Cardiospermum helicacabum, Terminalia arjuna and Aspergillus fumigatus (Gangadevi and Muthumary, 2008; Gangadevi and Muthumary, 2009; Kumar et al., 2019; Strobel et al., 1996a; Strobel et al., 1996b; Vennila et al., 2010). Furthermore, there are several anticancer compounds isolated from endophytic fungi following paclitaxel, but no of them has had such effect on cancer chemotherapy as paclitaxel (Kharwar et al., 2011).

Chitosan is a natural amino polysaccharide of $D$ glucosamine (GICN) and N-acetyl-D-glucosamine (GlcNAc) (No et al., 1989) and is a deacetylated distinctive form of chitin. Chitosan is characterized by polycationic, non-toxic, biodegradable and antimicrobial properties with numerous economic applications, especially in food, pharmaceutics and cosmetics (Morin-Crini et al., 2019). Chitosan was produced commercially from the exoskeleton of crustaceans through insensitive chemical treatments. However, this is the main drawback in the chitosan extraction process and has limitations in industrial applications due to limited crustacean shells supply, expensive production process and inconsistent physicochemical characteristics (Wu et al., 2005). These drawbacks nominate fungi to be a potentially promising source for chitosan production, as production and purification of chitosan from the fungal walls grown under controlled conditions offer a great advantage of being environmentally friendly and provides potential aspects for a reliable product (Dhillon et al., 2013b; Abdel-Gawad et al., 2017). As mentioned above, fungi are characterized by a fast growth with high cell biomasses, obtained by simple fermentation and cost-competitive processes, regardless of geographical location or season (Teng et al., 2001).

The current study aimed to 1) isolate the endophytic fungus Neopestalotiopsis clavispora and investigate its capability for paclitaxel production from fungal culture. 2) maximize the economic importance of paclitaxel through the concomitant production of fungal chitosan from the fungal biomass residue. 3) outlined paclitaxel anticancer activity and cytotoxicity against cancer cell lines.

\section{Materials and methods}

\subsection{Endophytic fungus isolation and identification}

Avocado fruits (Persea americana L.) (10 samples) were collected from a market in Makkah city, Saudi Arabia in sterile polyethylene bags and transferred to the laboratory. One centimeter square from avocado fruits was washed by distilled water and surface sterilized using ethanol (70\%) for $1 \mathrm{~min}$ and sodium hypochlorite (5\%) for $3 \mathrm{~min}$. the pieces were rinsed subsequently and transferred to agar plates containing potato dextrose agar medium (potato, $200 \mathrm{~g} / \mathrm{l}$; dextrose, $20 \mathrm{~g} / \mathrm{l}$; agar, $20 \mathrm{~g} / \mathrm{l}$, distilled water, 1000 $\mathrm{ml}$ ) supplemented with chloramphenicol $(250 \mathrm{mg} / \mathrm{l})$ (Smith and Dawson, 1944). The plates were incubated at 28 ㅇ for 7 days. The growing fungal isolate was subcultured in pure culture and kept on a PDA slant at $4^{\circ} \mathrm{C}$ for further studies. Microscopic slides were prepared and examined under a light microscope (Olympus CX 41 microscope) equipped with a digital camera (SC30 Olympus digital camera, Japan) for morphological identification.

The molecular identification of isolated fungus was performed in SolGent Company, Daejeon, South Korea by amplification of nuclear ribosomal RNA using primers ITS1 (5' - TCC GTA GGT GAA CCT GCG G - 3') and ITS4 (5'- TCC TCC GCT TAT TGA TAT GC $\left.-3^{\prime}\right)$. The sequence of $18 \mathrm{~S}$ rRNA of a fungal strain containing 501 bases was first analyzed using the advanced BLAST search program on the NCBI website: http://www.ncbi.nlm.nih.gov/BLAST/T in order to assess the degree of DNA similarity. The nucleotide sequence of fungal strain ASU1 was deposited in the Genbank nucleotide sequence database under accession number KY624416. Phylogenetic tree derived from $18 \mathrm{~S}$ rRNA gene sequence was generated using phylogeny.fr software (http://www.phylogeny.fr/) in comparison to 18SrRNA gene sequences from eight different standard fungal strains obtained from Genbank: Phoma sp. QC2014b (KP330455), Phoma herbarum (EU754186), Fusarium oxysporum f. sp. cepae (GU166124), Penicillium janthinellum (GU981625), Pestalotiopsis paeoniicola (EU400221), Neopestalotiopsis clavispora (KR052094), Pestalotiopsis sp. LK2 (EU047942), Neopestalotiopsis foedans (KU593530) (Abdel-Sater et al., 2016).

\subsection{Paclitaxel production by endophytic fungus}

\subsubsection{Fungal inoculums preparation}

Neopestalotiopsis clavispora ASU1, isolated from Avocado fruits and maintained in PDA (potato dextrose agar) at $4{ }^{\circ} \mathrm{C}$, was used in this study. Spore suspension was used for inoculation of the flask cultures. Potato dextrose agar plates were inoculated with fungal spores and incubated for 7 days at $28 \pm 1{ }^{\circ} \mathrm{C}$. After growth, fungal hyphae were collected and blended with sterilized distilled water. One $\mathrm{ml}$ of the homogenous solution of fungal propagules was used for inoculation.

2.2.2. Culture medium and cultivation method for paclitaxel production 
The flask culture sucrose malt medium contained $(\mathrm{g} / \mathrm{l})$ : Sucrose, 50; malt extract, 17; peptone, 3; and chloramphenicol, 0.25. After sterilization, the medium was inoculated with $1 \mathrm{ml}$ of spore suspension. $250 \mathrm{ml}$ Erlenmeyer flasks containing $100 \mathrm{ml}$ of sterilized medium were incubated on a rotary shaker (Environ 3597-1, Labline Instruments, USA) at $120 \mathrm{rpm}$ at $28^{\circ} \mathrm{C}$ for 10 days in the dark.

\subsubsection{Extraction of paclitaxel from fungal culture}

Neopestalotiopsis clavispora ASU1 was grown in submerged culture as described above, after 10 days the culture (medium-plus mycelium) was extracted twice with an equal volume of $95 \%$ methanol (Strobel et al., 1996b). The filtrate with organic solvent was shacked over the night and dried in an evaporator under a vacuum at $65^{\circ} \mathrm{C}$. The residue was dissolved in $1 \mathrm{ml}$ methanol and analyzed by HPLC. Furthermore, the fungal biomass residue was collected and dried at $50^{\circ} \mathrm{C}$ up to constant weight and used for chitosan production.

\subsection{Characterization of fungal paclitaxel}

\subsubsection{High-performance liquid chromatography (HPLC)}

The extracted fungal paclitaxel was analyzed using Agilent HPLC (Agilent Technologies Series 1200, G1315DDAD, at the Analytical Chemistry Unit, Chemistry Department, Faculty of Science, Assiut University). With column Zorbax Extend $\mathrm{C} 18$, analytical (4.6 $\times 150 \mathrm{~mm}-5$ Micron) and mobile phase isocratic elution: A: methanol $70 \%$, B: milli-Q $30 \%$. The flow rate was $1.5 \mathrm{ml} / \mathrm{min}$ at $30^{\circ} \mathrm{C}$. The injection volume was $100 \mu \mathrm{L}$ and the detector was DAD at $232 \mathrm{~nm}$ (Markeb et al., 2016).

\subsubsection{Liquid chromatography-MS-MS (LC/MS-MS)}

After HPLC analysis of the extracted fungal paclitaxel, chromatographic analysis by LC/MS-MS was also performed using Agilent LC/Ms (Agilent Technologies 6420 Triple Quad LC/Ms, at the Analytical Chemistry Unit, Chemistry Department, Faculty of Science, Assiut University) for confirmation of paclitaxel concentration. 5 $\mu \mathrm{l}$ of fungal paclitaxel was injected into an Agilent LC/MS Triple Quad LC/Ms equipped with Zorbax Eclipse XDB-C18, analytical column (4.6 $\times 150 \mathrm{~mm}-5$ Micron) and mobile phase B: Milli-Q elution $10 \%$, C: Methanol $70 \%$. The flow rate was $0.8 \mathrm{ml} / \mathrm{min}$ at $40{ }^{\circ} \mathrm{C}$. Ms QQQ mass spectrometer system was operated as follows: The ion source is electrospray (ESI) positive ion mode; the gas temperature was $350{ }^{\circ} \mathrm{C}$ and the gas flow rate was set at $11 \mathrm{l} / \mathrm{min}$; nebulizer was $45 \mathrm{psi}$ and voltage setting on the capillary with the ion spray voltage was controlled at $4 \mathrm{kV}$. Paclitaxel (Sigma Aldrich) was measured and used as a standard (ElMaali et al., 2018).

\subsubsection{Fourier transforms infrared spectroscopy (FT-IR)}

Spectroscopic investigations on paclitaxel samples are of importance in the present and studied extensively by many scientists (Schmitt et al., 2015). The infrared spectrum of a compound is the superposition of the absorption bands of specific functional groups and can be used as a fingerprint for unknown compounds identification in comparison with recorded reference spectra. To detect the functional groups of paclitaxel in the fungal extract, powders of paclitaxel were tested by the FTIR method using a spectrophotometer model was analyzed using the $\mathrm{KBr}$ pressed disk technique (Thermo Scientific Nicolet iS10 FTIR Spectrometer) at Chemistry Department, Faculty of Science, Assiut University.

\subsubsection{MTT cell viability assay}

Effect of methanol and chloroform extracted paclitaxel from fungal culture on MCF7 breast cancer cells viability and toxicity were compared to that of pure taxol were investigated using 3-(4,5-dimethylthiazol-2-yl)-2,5diphenyltetrazolium bromide (MTT) MTT cell viability assay as previously described (Ismail et al., 2013). In brief, about $1 \times 10^{4}$ cells/well were cultured in 96-well plates and then incubated for $24 \mathrm{~h}$. On the following day, different concentrations of extracted paclitaxel (methanol and chloroform) from the culture medium were added to the cells for $24 \mathrm{~h}$ and positive controls (methanol and chloroform) were involved in the experimental design to avoid their cytotoxic effect on the cells. As well, pure taxol (purchases taxol) was applied to compare the cytotoxic activity of extracted paclitaxel and pure taxol. Next, fifty $\mu \mathrm{l}$ of MTT solution ( $2 \mathrm{mg} / \mathrm{ml}$ of MTT in PBS) were added to each well and left for $4 \mathrm{~h}$, then the supernatants were removed carefully and $150 \mu$ DMSO were added to each well. The 96-well plates were shaken for 10 minutes and then were read at $A 570$ with a reference filter at $A 650$ using an ELISA plate reader (Ismail et al., 2013).

\subsubsection{Chitosan extraction from fungal biomass}

After fungal cultivation on sucrose malt medium, the culture (fungal biomass and medium) was used for paclitaxel production, then filtered by filter paper (Whatman No.1) and the fungal biomass residue was used for chitosan production. Dried fungal biomass was homogenized with $0.5 \mathrm{~N}$ sodium hydroxide solution (1:30, $\mathrm{w} / \mathrm{v})$ at $121^{\circ} \mathrm{C}$ for $20 \mathrm{~min}$ for the deproteinization process. The alkali insoluble materials (AIM) were centrifuged at $10000 \mathrm{rpm}$ for $15 \mathrm{~min}$ and subsequently washed with distilled water until the $\mathrm{pH}$ was completely neutralized, dried and weighted. Then, 1 gram of dried AIM was treated with $40 \mathrm{ml}, 2 \%$ acetic acid at $95^{\circ} \mathrm{C}$ for $6 \mathrm{~h}$ (Synowiecki and Al-Khateeb, 1997). The extract containing fungal chitosan was isolated by centrifugation at $10000 \mathrm{rpm}$ for $15 \mathrm{~min}$, the supernatant containing the chitosan was collected, adjusting $\mathrm{pH}$ to 10.0 with $2 \mathrm{M} \mathrm{NaOH}$ followed by centrifugation at 10,000 rpm for $15 \mathrm{~min}$ for separation of chitosan. The precipitation was washed with distilled water until reaching $\mathrm{pH} 7$, followed by $95 \%(\mathrm{v} / \mathrm{v})$ ethanol $(1: 20$, $\mathrm{w} / \mathrm{v})$. Finally, dried at $60^{\circ} \mathrm{C}$ for $24 \mathrm{~h}$. Fungal chitosan (virtually pure mycelial chitosan) yield was calculated by weight (g). The alkali-insoluble precipitate obtained after suitable alkali and acid extraction contained nearly pure chitosan (McGahren et al., 1984). The crude chitosan yield of residual fungal biomass was calculated from the following equation (Abdel-Gawad et al., 2017):

Chitosan yield $(\%)=[$ dry wt. of obtained chitosan /dry wt. of sample] $\times 100$

\subsection{Characterization of fungal Chitosan}

\subsubsection{Determination of glucosamine content}


Fungal chitosan samples were hydrolyzed with $2 \mathrm{M}$ hydrochloric acid at $110{ }^{\circ} \mathrm{C}$ for $2 \mathrm{~h}$ and the liberated $\mathrm{D}$ glucosamine was determined by dinitrosalicylic acid according to Huet et al. (2006).

\subsubsection{Infra-red spectroscopy}

The structure of extracting mycelial chitosan was confirmed by infrared spectroscopy using a $\mathrm{KBr}$ pellet method in FTIR (Thermo Scientific Nicolet iS10 FT-IR Spectrometer) at Chemistry Department, Faculty of Science, Assiut University. FTIR spectra were recorded in the middle infrared $\left(4000 \mathrm{~cm}^{-1}\right.$ to $\left.500 \mathrm{~cm}^{-1}\right)$.

\subsubsection{Degree of deacetylation}

The degree of deacetylation (DD) was determined according to the baseline method (Khan et al., 2002). The IR spectrum recording procedure is the same as described above. According to the IR spectrum, the DD was calculated by measuring the absorbance ratio of A1655 and A3450 (Baxter et al., 1992). The amide I band at $1655 \mathrm{~cm}^{-1}$ and the hydroxyl group absorption band at $3450 \mathrm{~cm}^{-1}$ were used as an internal reference, DD was assessed based on the following equation:

$$
\begin{aligned}
\text { Degree of deacetylation }= & 100-[(\text { A1655/A3450) } \\
& \times 115]
\end{aligned}
$$

\subsubsection{Determination of antioxidant activity by reducing power measurement}

The reducing power of chitosan extract was determined by the method of Chang et al., (2002) with some modification. An aliquot of $0.5 \mathrm{ml}$ chitosan sample $(5: 100 \mathrm{w} / \mathrm{v})$, in $0.2 \%$ acetic acid solution was added to $0.1 \mathrm{ml}$ of $1 \%(\mathrm{w} / \mathrm{v})$ potassium ferricyanide. The mixture was incubated at $50{ }^{\circ} \mathrm{C}$ for $30 \mathrm{~min}, 0.1 \mathrm{ml}$ of trichloroacetic acid $(1 \% \mathrm{w} / \mathrm{v})$ and 0.1 $\mathrm{FeCl}_{3}(0.1 \% \mathrm{w} / \mathrm{v})$ were added and left for $20 \mathrm{~min}$. The result was recorded by measuring the amount of ferric ferrocyanide (Prussian blue) formed using spectrophotometer at $700 \mathrm{~nm}$ and ascorbic acid was used as a positive control.

\section{Results}

\subsection{Morphological and molecular characterization of the fungus}

The endophytic fungus isolated from avocado fruits was identified morphologically and genetically as Neopestalotiopsis clavispora. The isolate is characterized morphologically by dark brown, subglobose conidioma; conidia with 4 transverse septa, fusiform to clavate, smooth-walled, hyaline, 20-27 $\mu$ m length (Judith-Hertz, 2016) as shown in Figure 1. Morphological features of the fungus were confirmed by $18 \mathrm{~S}$ rRNA sequence analysis (Maharachchikumbura et al., 2014). A partial 18S rRNA gene sequence of approximately 501 base pairs of fungal strain ASU1 has a sequence with $99 \%$ similarity with Neopestalotiopsis clavispora (KR052094), N. foedans (KU593530) and Pestalotiopsis paeoniicola (EU400221) that is available in the Genbank database. A phylogenetic tree was constructed from multiple sequence alignment of $18 \mathrm{~S}$ rRNA gene sequences (Figure 2). The nucleotide sequence of fungal strain ASU1 was deposited in Genbank with accession number KY624416.

\section{A}

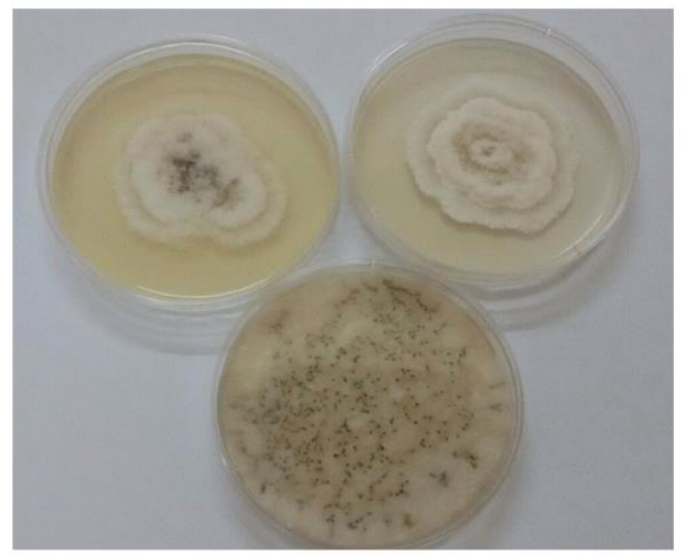

B

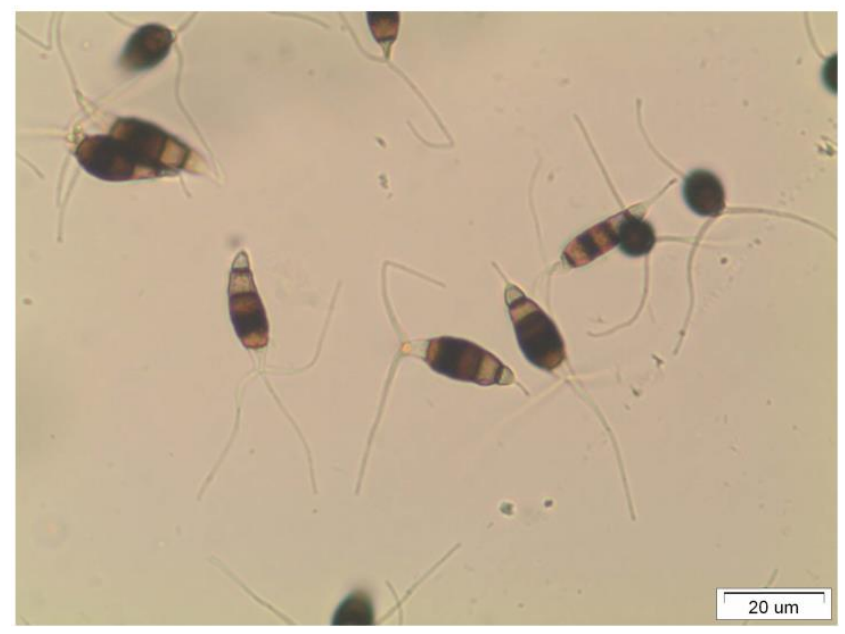

Figure 1. Image of Neopestalotiopsis clavispora growth on PDA medium (A), Neopestalatiopsis conidia with 4 transverse septa, fusiform to clavate, and smooth-walled (B).

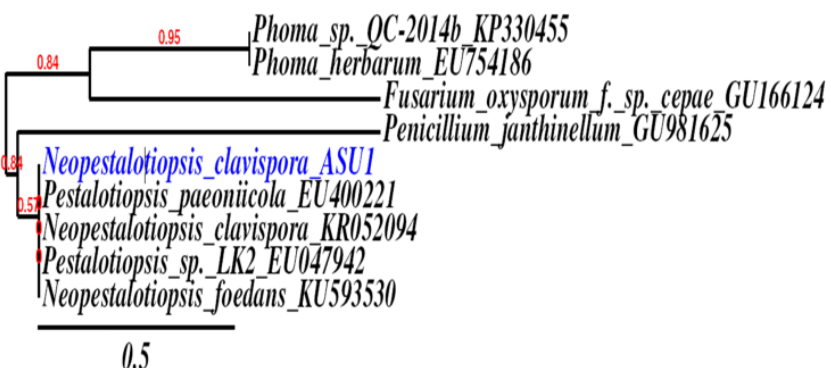

Figure 2. Phylogenetic tree of Neopestalotiopsis clavispora ASU1 (KY624416).

\subsection{Production of paclitaxel}

In the present study, Neopestalotiopsis clavispora KY624416 was grown on malt sucrose medium with a higher growth rate recording $14.2 \pm 0.095 \mathrm{~g} / \mathrm{l}$ dry biomass with potentiality for anticancer compounds production. HPLC analysis of paclitaxel produced by endophytic fungus Neopestalotiopsis clavispora KY624416 elucidated that fungal strain could produce anticancer compounds in liquid medium recording a single peak identical with the retention time of authentic paclitaxel (Figure 3). Although a defined identical single peak was produced from HPLC 
analysis, it may contain other taxanes (Figure 3). The quantitative analysis of paclitaxel and related taxanes was performed with LC/MS-MS that may be very important to discriminate paclitaxel from other closely related taxanes. In the present study, it was confirmed that the LC/MS chromatographic analyses are highly accurate showing that Neopestalotiopsis clavispora KY624416 paclitaxel extract, had four peaks at $\mathrm{m} / \mathrm{z}$ from 876.7 to 877.7 , that similar to the peak obtained with authentic paclitaxel (Figure 4). LC/Ms-Ms analysis of fungal paclitaxel confirmed that fungal strain could produce paclitaxel with concentration recording $100.6 \mu \mathrm{g} / \mathrm{l}$.

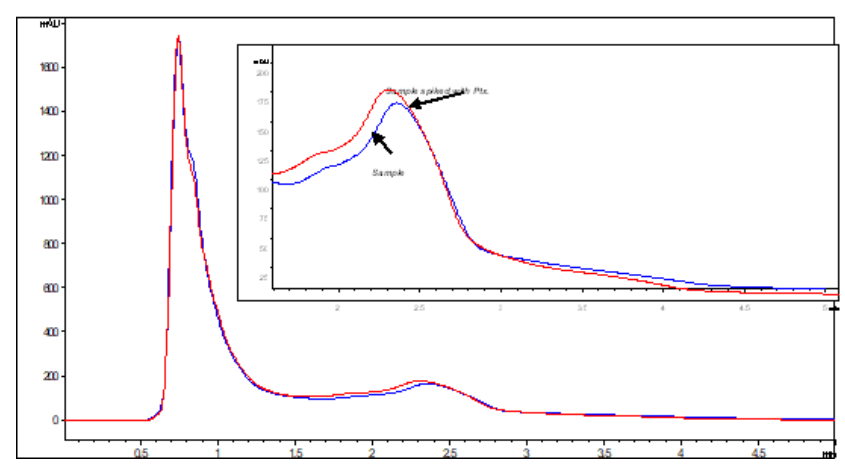

Figure 3. HPLC chromatogram of standard taxol and fungal paclitaxel isolated from Neopestalotiopsis clavispora ASU1 (KY624416).

\subsection{FT-IR analysis}

The resultant absorption spectrum indicates the presence of various chemical bonds and functional groups present in the sample. The Fourier transform infrared spectroscopy (FT-IR) analysis measures the selective absorption of light by the vibration modes of specific chemical bonds in the sample determining several functional groups such as $\mathrm{OH}$ hydroxyl and amide $(-\mathrm{NH})$ groups stretch $\left(3392.65 \mathrm{~cm}^{-1}\right)$, aliphatic $\mathrm{CH}$ stretch $\left(2900 \mathrm{~cm}^{-1}\right)$, aromatic ring $\mathrm{C}=\mathrm{C}$ stretch was positioned at $1652.61 \mathrm{~cm}^{-1}, \mathrm{NH}$ stretching (1455.93 $\mathrm{cm}^{-1}$ ), COO stretching frequency was observed at 1245.11 $\mathrm{cm}^{-1}$ and aromatic $\mathrm{C}$ and $\mathrm{H}$ bend $\left(1082.52 \mathrm{~cm}^{-1}\right)$ as shown in Figure 5.

\subsection{Cell viability}

Cell viability was investigated in MCF-7 breast cancer cell line after $24 \mathrm{~h}$ of treatment with different extracted (methanol, chloroform) paclitaxel concentrations (0.0, $0.01,0.05,0.1,0.15$, and $0.3 \mu \mathrm{M}$ ) and compared with purchases taxol using MTT assay. Quantitatively, it was shown that taxol drug exerted a dose-dependent cytotoxic effect. Statistically, the significant cytotoxic effect was $(P<$ $0.05)$ at 10,50 and $100 \mathrm{nM},(P<0.01)$ at $150 \mathrm{nM}$ and $(P<$ $0.001)$ at $300 \mathrm{nM}$ while the extracted fungal paclitaxel exerted a significant cytotoxic effect $(P<0.05)$ only at 300 $\mathrm{nM}$. Regarding the comparison among fungal taxol, our results revealed that the methanol extract was the highly effective compared to the other tested extracts which exerted more cytotoxicity at nearly all concentrations. Statistically, the differences among fungal taxol showed no significant cytotoxic effect on MCF-7 breast cancer cells (Table 1).
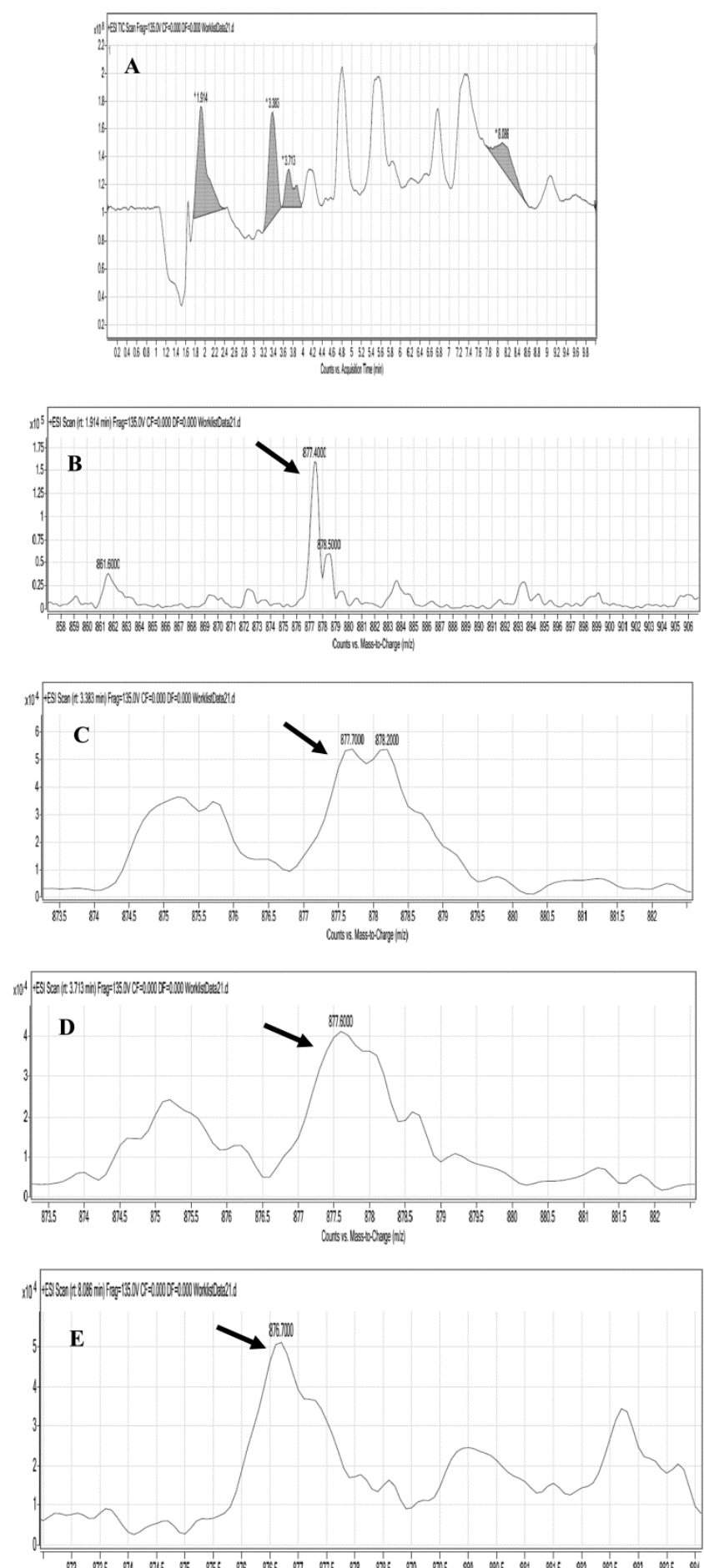

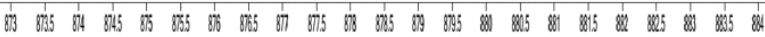

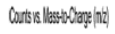

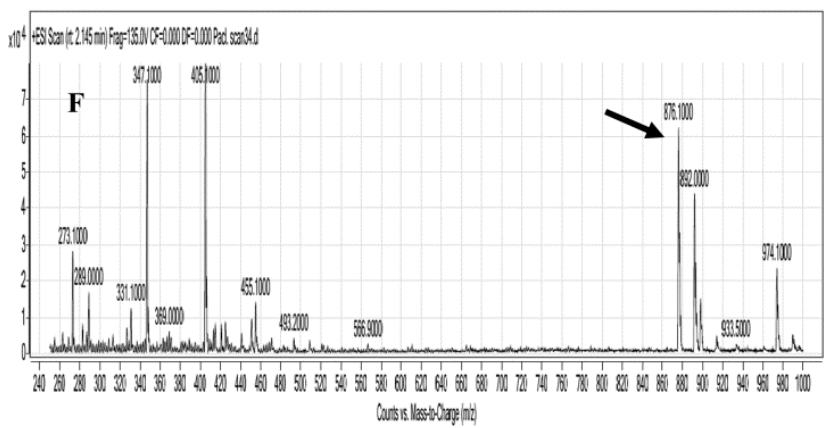

Figure. 4. LC/Ms chromatograms showing fungal paclitaxel scan (A) with four peaks at $\mathrm{m} / \mathrm{z}$ from 876.7 to 877.7 (B-F), that similar to the peak obtained with authentic paclitaxel $(E)$. 


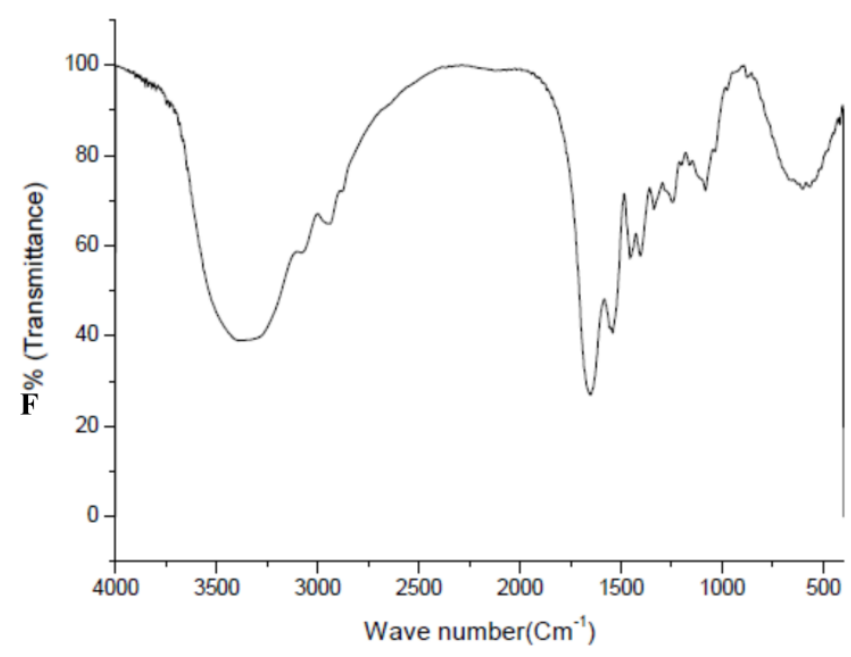

Figure 5. FT-IR spectrum of paclitaxel produced by $N$ eopestalotiopsis clavispora ASU1.

\subsection{Chitosan production from fungal biomass wastes}

The results obtained showed that the chitosan yield from fungal biomass represented $5.94 \%$ of fungal dry biomass glucosamine content of extracted fungal chitosan was estimated by 3, 5 dinitrosalysilic acid reagent yielding 73.53 $\%$ of extracted chitosan (Figure 6).

\subsubsection{Characterization of fungal chitosan}

\subsubsection{FT-IR analysis}

Chitosan extracted from residual fungal biomass after the fermentation process for paclitaxel production was subjected to FT-IR analysis. The FT-IR spectra in Figure 7 showed typical absorption bands of chitosan: the $\mathrm{OH}$ stretching band at $3427.6 \mathrm{~cm}^{-1}$ the stretching vibrations overlapped with $\mathrm{N}-\mathrm{H}$ stretching band; the $\mathrm{C}-\mathrm{H}$ band within $2924.51 \mathrm{~cm}^{-1}$. The band identified as an amine group (N-H bending bands) absorbed infrared between $1651.4 \mathrm{~cm}^{-1}$ $1566.7 \mathrm{~cm}^{-1}$. The skeletal vibrations involving the C-O-C stretching band at 1035.57; the $-\mathrm{CH}_{2}$ bending centered at $1416.49 \mathrm{~cm}^{-1}$. Also, the spectra showed the amide bonds at approximately 1651.40 and $11566.73 \mathrm{~cm}^{-1}$; amide I band at 1651.4 and amide II band at $1566.73 \mathrm{~cm}^{-1}$. The IR spectroscopic method is used to determine the degree of deacetylation (DD) value of mycelial chitosan. The DD value of mycelial chitosan obtained from submerged fermentation of Neopestalotiopsis clavispora was $54.60 \%$ (Figure 6).

Table 1: Mean values \pm SD of absorbance (MTT assay) and cell viability percentage in breast cancer cell line (MCF7) after $24 \mathrm{hr}$ of different doses of different extracted taxol treatment

\begin{tabular}{ccccccc}
\hline \multirow{2}{*}{ Treatment } & \multirow{2}{*}{ Standard taxol (purchases taxol) } & \multicolumn{4}{c}{ Fungal paclitaxel } \\
\cline { 3 - 7 } & & & \multicolumn{2}{c}{ Methanol extract } & \multicolumn{2}{c}{ Chloroform extract } \\
\hline Dose & Mean \pm SD & Viability\% & Mean \pm SD & Viability\% & Mean \pm SD & Viability\% \\
\hline $0 \mathrm{nM}$ & $0.76 \pm 0.07$ & 100 & $0.56 \pm 0.03$ & 100 & $0.50 \pm 0.007$ & 100 \\
\hline $10 \mathrm{nM}$ & $0.59 \pm 0.05^{\mathrm{a}}$ & 77.2 & $0.51 \pm 0.1$ & 87.9 & $0.44 \pm 0.056$ & 87.3 \\
\hline $50 \mathrm{nM}$ & $0.51 \pm 0.06^{\mathrm{a}}$ & 66.5 & $0.49 \pm 0.02$ & 85.2 & $0.42 \pm 0.066$ & 83.4 \\
\hline $100 \mathrm{nM}$ & $0.45 \pm 0.03^{\mathrm{a}}$ & 58.2 & $0.48 \pm 0.1$ & 81.9 & $0.41 \pm 0.038$ & 80.6 \\
\hline $150 \mathrm{nM}$ & $0.33 \pm 0.05^{\mathrm{b}}$ & 43.3 & $0.46 \pm 0.12$ & 78.1 & $0.40 \pm 0.039$ & 78.6 \\
\hline $300 \mathrm{nM}$ & $0.2 \pm 0.03^{\mathrm{bc}}$ & 26.7 & $0.44 \pm 0.11^{\mathrm{a}}$ & 74.5 & $0.38 \pm 0.020^{\mathrm{a}}$ & 75.5 \\
\hline
\end{tabular}

\subsubsection{Antioxidant activity of chitosan}

The antioxidant activity of chitosan extracted from Neopestalatiopsis clavispora waste biomass was studied using ferric reducing antioxidant activity recording $0.263 \pm 0.051 \mathrm{mg} / \mathrm{ml}$, this result revealed that fungal chitosan has potent reducing power and antioxidant power.

\section{Discussion}

\subsection{Production of paclitaxel}

Currently, most of the paclitaxel produced for medical uses is synthesized by a chemical semi-synthetic approach (Holton et al., 1995). However, the extraction of taxol from Taxus plants is relatively complex with low yields, depending on epigenetic, seasonal and environmental conditions (Jiang et al., 2021; Zhu and Chen 2019) . In the eighteenth century, endophytic fungi become a useful tool for the paclitaxel production process of the future, great progress in this field have been obtained. However, despite its many advantages as, shorter generation time, higher growth rate, easily genetic manipulation, low substrate costs for endophytic fungi growth and easy recovery of paclitaxel from the fermentation medium; some impediments are resulting from yields and titers remain low compared with that of the taxes trees (Demain and Solomon, 1981; Stierle et al., 1993).

In the present study, the endophytic fungus isolated from avocado fruits was identified based on morphological and genetic analysis as Neopestalotiopsis clavispora and was deposited in Genbank with accession number KY624416. N. clavispora KY624416 showed a high potentiality for paclitaxel production, an anticancer compound which was analyzed by HPLC and LC/MS-MS analysis recording 100.6 $\mu \mathrm{g} / \mathrm{I}$. In this respect, Pestalotiopsis microspora isolated from the inner bark of Himalayan yew could produce taxol in mycelial culture (Strobel et al., 1996b). Paclitaxel was originally produced from the Taxus plant, it was reported that the gene responsible for paclitaxel production may transfer from the plant to corresponding endophytic fungi (Stierle et al., 1993). These fungi may produce paclitaxel for competition and survive inside their hosts (Young et al., 1992). On the other hand, paclitaxel biosynthetic gene candidates in endophytic fungus Penicillium aurantiogriseum NRRL 62431 are quite different from those in hosts and they provided evidence that horizontal gene transfer is unlikely to have occurred. Several 
endophytic fungi from different host plants have been investigated for their ability for paclitaxel production (Mirjalili et al., 2012; Yang et al., 2014). In our study we observed paclitaxel production by endophytic fungus Neopestalotiopsis clavispora KY624416 during this batch fermentation process is quite good in comparison with various endophytic fungi reported in the literature.

FT-IR analysis for extracted paclitaxel sample showed absorption spectrum and functional groups such as $\mathrm{OH}$ hydroxyl and amide (-NH) groups stretch, aliphatic $\mathrm{CH}$ stretch, aromatic ring $\mathrm{C}=\mathrm{C}$ stretch, $\mathrm{NH}$ stretching, $\mathrm{COO}$ stretching frequency and aromatic $\mathrm{C}$ and $\mathrm{H}$ bends. The observation of the vibration spectrum of paclitaxel in comparison with standard taxol spectrum (Raj et al., 2015; Vennila and Muthumary, 2011). Consequently, FT-IR spectroscopy was widely used recently by researchers to verify the chemical characteristics of paclitaxel in comparison with standard available spectrum (Hiremath et al., 2013; Kathiravan et al., 2014; Schmitt et al., 2015).

\subsection{Cell viability}

From cell viability results using MCF-7 breast cancer cell line, it was found that the most effective one of paclitaxel extracts is the methanol extract which exerted more cytotoxicity at nearly all concentrations. As well, the IC50 values of strong cytotoxic activity of commercial taxol, methanol extract and chloroform extract were found against MCF-7 breast cancer cell line at $158.1 \mathrm{nM}, 621.7$ $\mathrm{nM}, 667.3 \mathrm{nM}$ and $596.4 \mathrm{nM}$, respectively. As well, fungal paclitaxel is a proven anti-cancer agent that has been tested in various human carcinoma cell lines, such as MCF7 (Kumaran et al., 2012; Pandi et al., 2011), BT220 (Gangadevi and Muthumary, 2007; Kumaran et al., 2012), HL251 (Kumaran et al., 2012), Hep G2 cell lines (Rajendran et al., 2013). Supporting the earlier findings that at low concentration taxol could inhibit cell proliferation by blocking mitosis (Gangadevi and Muthumary, 2007; Kumaran et al., 2012). Also, Cladosporium oxysporum taxol showed cytotoxic activity towards HCT 15 cancer cell line for $24 \mathrm{~h}$ treatment with an IC50 value of $3.5 \mu \mathrm{M}$ concentration (Raj et al., 2015). In conclusion, fungal endophytes are gaining importance because of their potentiality to produce medically important bioactive compounds.

\subsection{Chitosan production from fungal biomass residue}

The chitosan yield extracted from fungal biomass wastes represented $5.94 \%$ of fungal dry biomass and the glucosamine content of extracted fungal chitosan was $73.53 \%$ of extracted chitosan (Figure 6). Chitosan yield by endophytic fungus Neopestalotiopsis clavispora KY624416 grown on sucrose malt broth medium is quite good when compared with values using various fungi using different substrates reported in the literature in this instance. Chitosan was extracted from different fungal species such as, Cunninghamella elegans $(0.75 \mathrm{~g} / \mathrm{l}), \mathrm{C}$. blakesleeana $(9.4$ $\%$ of fungal biomass), Rhizomucor miehei (13.67 \%), Mucor rouxii (1.2\%) Cladosporium Cladosporioides (25.2 mg/g) with degree of deacetylation $81 \%, 35 \%, 80.6 \%$ and $59 \%$, respectively (George et al., 2011; Miyoshi et al., 1992;
Tajdini et al., 2010; Tayel et al., 2016). As well as the extracted chitosan of some fungi was tested for their biotechnological applications in water pollutant elimination (heavy metals and waterborne microorganisms) and removal of toxic pollutants biocontrol of postharvest diseases (Betchem, et al., 2019; CortésRivera et al., 2021) ). Furthermore, these results provide a promising approach for concomitant production of paclitaxel and chitosan sources and increase the possibility for production and extraction of fungal chitosan with welldefined properties at an industrial scale.

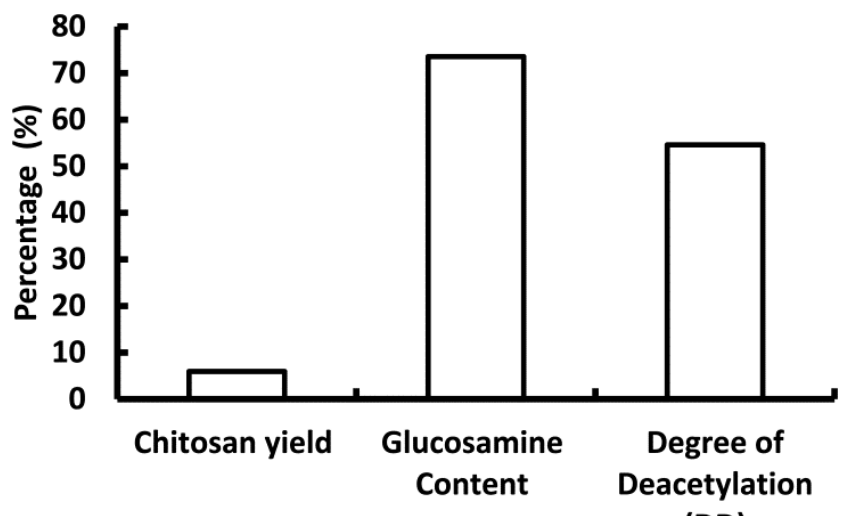

(DD)

Figure 6. Percent of chitosan yield from fungal biomass residue after paclitaxel extraction, glucosamine content in extracted chitosan and the degree of deacetylation.

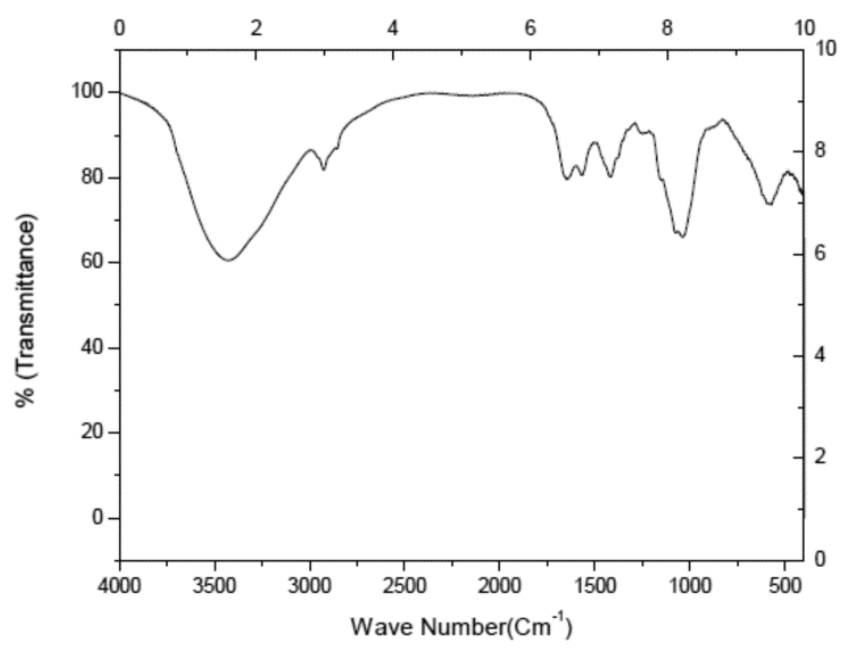

Figure 7. FT-IR spectrum of chitosan extracted from fungal biomass.

IR spectroscopy of chitosan extracted from residual fungal biomass provided a relatively quick, simple method used for the qualitative and quantitative determination of chitin and chitosan characteristics, mainly specific absorption groups present in the recovered product, the degree of acetylation/deacetylation and chitosan impurities (Baxter et al., 1992). The FT-IR spectra showed absorption bands; the $\mathrm{OH}$ stretching band, the $\mathrm{C}-\mathrm{H}$ band, an amine group ( $\mathrm{N}$ $\mathrm{H}$ bending bands) as recorded by Pavia et al., (2014). As well as, the skeletal vibrations include the $\mathrm{C}-\mathrm{O}-\mathrm{C}$ stretching band; the $-\mathrm{CH}_{2}$ bending (Kaya et al., 2015; Kumirska et al., 2010; Negrea et al., 2015). Also, the spectra showed the amide bonds; amide I band at 1651.4 and amide II band. 
The current results were in harmony with the findings of Kasaai et al., (2000); Khan et al., (2002); Also, FT-IR analysis of chitosan obtained from the mycelia of Basidiomycetes exhibited several bands similar with internal reference spectra of chitosan such as amide bonds at approximately 1655 and $1313 \mathrm{~cm}^{-1}$ (Di Mario et al., 2008). In the current study, other functional groups included hydroxyl stretching band at $3427 \mathrm{~cm}^{-1}$, amide I band at 1655, primary amine band at $1638-1561 \mathrm{~cm}^{-1}$ amide II band at $1561 \mathrm{~cm}^{-1}$ and amide III bands at $1320 \mathrm{~cm}^{-1}$. These absorption bands preliminary approved the fungal extracted product to be chitosan.

The IR spectroscopic method is used to determine the degree of deacetylation (DD) value of mycelial chitosan. The degree of deacetylation of chitosan extracted from Neopestalotiopsis clavispora was $54.60 \%$ which is considered as one of the most important chemical characteristics influencing solubility, chemical reactivity and biodegradability, which in accordance influence the performance of chitosan in many applications (AbdelSalam, 2013; Morin-Crini et al., 2019) . Interestingly, these desirable physicochemical properties of fungal chitosan could increase the potential application for food, cosmetic, chemical and pharmaceutical industries. Consequently, fungal chitosan fermentative production is considered a good alternative to minimize environmental pollution from traditional chitosan production by strong alkali, furthermore increase the feasibility of a new, simple and green technology for the production of value-added chitosan (Dhillon et al., 2013a).

\subsection{Antioxidant activity of chitosan}

The antioxidant activity of chitosan produced from Neopestalatiopsis clavispora was evaluated using ferric reducing antioxidant activity (Zimoch-Korzycka et al., 2016). Hydroxyl radicals display a small diffusion capacity and high reactivity in the generation of cellular damages. These injuries speed up the aging process causing cancer and numerous diseases (Kehrer and Klotz, 2015). So, removing $\mathrm{OH}$ radicals is important for antioxidant defense in cell and food systems. Reducing power measures the ability of a sample to act as an electron donor and therefore, reacts with free radicals converting them to more stable products and thereby terminate radical chain reaction (Park et al., 2004). Fungal chitosan reducing power activitiy was $0.263 \pm 0.051 \mathrm{mg} / \mathrm{ml}$, this result revealed that fungal chitosan has potent reducing power and its antioxidant power may be attributed to hydroxyl groups $(\mathrm{OH})$, and amine groups.

\section{Conclusion}

Recently, the production of paclitaxel by endophytic fungi has gained much attention due to the need for costcompetitive and alternative sources for taxol production from Taxus plants. The present paper provides a promising approach through integrating a system for paclitaxel production from malt sucrose medium by endophytic fungus Neopestalotiopsis clavispora with chitosan production from residual fungal mycelium wastes. Paclitaxel productivity by endophytic fungus
Neopestalotiopsis clavispora $(100.6 \mu \mathrm{g} / \mathrm{l})$ with a significant cytotoxic effect on MCF7 cell line revealed the possibility and potentiality of this fungal strain for paclitaxel industrial biotechnological processes. Furthermore, the available fungal mycelium wastes, resulting from the paclitaxel extraction process, represent a promising candidate for chitosan production as compared to traditional sources from crustacean shells. The remarkable findings of this study have been proven to be practically important in the development of paclitaxel production processes and enhancing low-cost chitosan production from the utilization of fungal mycelium wastes. Presently, the industries sustain losses for the safe disposal of wastes, which leads to an increase in the final production cost of the products so, this paper is expected to provide a competitive economic outcome for industrial concomitant of paclitaxel and chitosan production by endophytic fungi.

\section{Conflict of interest}

The authors declare that there is no conflict of interest.

\section{References}

Abdel-Gawad K.M., Hifney A.F., Fawzy M.A. and Gomaa M. (2017). Technology optimization of chitosan production from Aspergillus niger biomass and its functional activities, Food Hydrocolloids, 63, 593-601.

Abdel-Salam H.A. (2013). Evaluation of nutritional quality of commercially cultured Indian white shrimp Penaeus indicus, International Journal of Food Sciences, 2, 160-166.

Abdel-Sater M.A., Moubasher A.-A.H. and Soliman Z.S.M. (2016). Diversity of filamentous and yeast fungi in soil of citrus and grapevine plantations in the Assiut region, Egypt. Czech Mycology, 68.

Alexandre J., Hu Y., Lu W., Pelicano H. and Huang P. (2007). Novel action of paclitaxel against cancer cells: bystander effect mediated by reactive oxygen species, Cancer Research, 67, 3512-3517.

Baxter A., Dillon M., Taylor K.A. and Roberts G.A. (1992). Improved method for ir determination of the degree of $\mathrm{N}$ acetylation of chitosan, International Journal of Biological Macromolecules, 14, 166-169.

Betchem G., Johnson N.A.N and Wang Y. (2019). The application of chitosan in the control of post-harvest diseases: A review. Journal of Plant Diseases and Protection, 126(6), 495-507.

Brown D. (2003). Taxus: the genus Taxus. Preclinical and clinical studies of the taxanes, 387-435.

Chakravarthi B., Das P., Surendranath K., Karande A.A. and Jayabaskaran C. (2008). Production of paclitaxel by Fusarium solani isolated from Taxus celebica. Journal of Bioscience, 33, 259-267.

Chang L.-W., Yen W.-J., Huang S.C. and Duh P.-D. (2002). Antioxidant activity of sesame coat, Food Chemistry, 78, 347-354.

Cortés-Rivera H.J., González-Estrada R.R., Huerta-Ocampo J.A., Blancas-Benítez F.J. and Gutiérrez-Martínez P. (2021). Evaluación de quitosano comercial y extractos acuosos de mesocarpio de coco (Cocos nucifera L.) para el control de Rhizopus stolonifer aislado de guanábana (Annona muricata L.): Pruebas in vitro. TIP Revista Especializada en Ciencias Químico-Biológicas, 24. 
Croom Jr. E. (1995). Taxus for taxol and taxoids, Taxol: Sci. Appl., CRC Press, Boca Raton, 37-70.

Demain A.L. and Solomon N.A. (1981). Industrial microbiology, Scientific American, 245, 42-51.

Dhillon G.S., Kaur S., Brar S.K. and Verma M. (2013a). Green synthesis approach: extraction of chitosan from fungus mycelia, Critical Reviews in Biotechnology, 33, 379-403.

Dhillon G.S., Kaur S., Sarma S.J. and Brar S.K. (2013b). Integrated process for fungal citric acid fermentation using apple processing wastes and sequential extraction of chitosan from waste stream, Industrial Crops and Products, 50, 346-351.

Di Mario F., Rapana P., Tomati U. and Galli E. (2008). Chitin and chitosan from Basidiomycetes, International Journal of Biological Macromolecules, 43(1), pp.8-12.

Dodane V. and Vilivalam V.D. (1998). Pharmaceutical applications of chitosan, Pharmaceutical Sciences and Technology Today, 1, 246-253.

El-Maali N.A., Mohrram A.M., El-Kashef H. and Gamal K. (2018). Novel resources of Taxol from endophytic and entomopathogenic fungi: Isolation, characterization and LC-Triple mass spectrometric quantification, Talanta, 190, 466-474.

Frense D. (2007). Taxanes: perspectives for biotechnological production. Applied Microbiology and Biotechnology, 73, 1233-1240.

Gallego A., Malik S., Yousefzadi M., Makhzoum A., TremouillauxGuiller J. and Bonfill M. (2017). Taxol from Corylus avellana: paving the way for a new source of this anti-cancer drug. Plant Cell, Tissue and Organ Culture (PCTOC), 129(1), 1-16.

Gangadevi V. and Muthumary J. (2007). Preliminary studies on cytotoxic effect of fungal taxol on cancer cell lines, Afr. J Biotechnol, 6, 1382-1386.

Gangadevi V. and Muthumary J. (2008). Isolation of Colletotrichum gloeosporioides, a novel endophytic taxolproducing fungus from the leaves of a medicinal plant, Justicia gendarussa, Mycology Balcanica, 5, 1-4.

Gangadevi V. and Muthumary J. (2009). Taxol production by Pestalotiopsis terminaliae, an endophytic fungus of Terminalia arjuna (arjun tree), Biotechnology and Applied Biochemistry, 52, 9-15.

George T.S., Guru K.S.S., Vasanthi N.S. and Kannan K.P. (2011). Extraction, purification and characterization of chitosan from endophytic fungi isolated from medicinal plants, World Journal of Science and Technology, 1, 43-48.

Guenard D., Gueritte-Voegelein F. and Potier P. (1993). Taxol and taxotere: discovery, chemistry, and structure-activity relationships, Accounts of Chemical Research, 26, 160-167.

Heinig U., Scholz S. and Jennewein S. (2013). Getting to the bottom of Taxol biosynthesis by fungi, Fungal Diversity, 60, 161-170.

Hiremath J.G., Khamar N.S., Palavalli S.G., Rudani C.G., Aitha R. and Mura P. (2013). Paclitaxel loaded carrier based biodegradable polymeric implants: preparation and in vitro characterization, Saudi Pharmaceutical Journal, 21, 85-91.

Holton R.A., Biediger R.J. and Boatman P.D. (1995). Semisynthesis of taxol and taxotere, Taxol: science and applications. CRC Press, Boca Raton, FL, pp. 97-121.

Huet J., Wyckmans J., Wintjens R., Boussard P., Raussens V., Vandenbussche G., Ruysschaert J.M., Azarkan M. and Looze Y. (2006). Structural characterization of two papaya chitinases, a family GH19 of glycosyl hydrolases, Cellular and Molecular Life Sciences, 63, 3042-3054.
Ismail I.A., Kang H.S., Lee H.-J., Chang H., Yun J., Lee C.W., Kim N.H., Kim H.S., Yook J.I. and Hong S.-H. (2013). 2-Hydroxycinnamaldehyde inhibits the epithelial-mesenchymal transition in breast cancer cells, Breast Cancer Research and Treatment, 137, 697-708.

Jiang L., Zhang K., Lü X., Yang L., Wang S., Chen D., Yang Y. and Qiu D. (2021). Characterization and expression analysis of genes encoding Taxol biosynthetic enzymes in Taxus spp, Journal of Forestry Research, 1-9.

Judith-Hertz C. (2016). Sytematics and species delimitation in Pestalotia and Pestalotiopsis sl (Amphisphaeriales, Ascomycota), Ph.D. Thesis, Goethe -Universität, Frankfurt am Main.

Kasaai M.R. Arul J. and Charlet G. (2000). Intrinsic viscositymolecular weight relationship for chitosan, Journal of Polymer Science Part B: Polymer Physics, 38, 2591-2598.

Kathiravan G., Raman S.V., Rajangam B. and Rajasekar A. (2014). Infra-red spectral analysis of taxol produced by different species of Pestalotiopsis, Journal of Analytical \& Bioanalytical Techniques, 5, 1.

Kaya M., Baran T., Asan-Ozusaglam M., Cakmak Y.S., Tozak K.O., Mol A., Mentes A. and Sezen G. (2015). Extraction and characterization of chitin and chitosan with antimicrobial and antioxidant activities from cosmopolitan Orthoptera species (Insecta), Biotechnology and Bioprocess Engineering, 20, 168.

Kehrer J.P. and Klotz L.-O. (2015). Free radicals and related reactive species as mediators of tissue injury and disease: implications for Health, Critical Reviews in Toxicology, 45, 765-798.

Khan T.A., Peh K.K. and Ch'ng H.S. (2002). Reporting degree of deacetylation values of chitosan: the influence of analytical methods, Journal of Pharmaceutical Sciences, 5, 205212.

Kharwar R.N., Mishra A., Gond S.K., Stierle A. and Stierle D. (2011). Anticancer compounds derived from fungal endophytes: their importance and future challenges, Natural Products Reports, 28, 1208-1228.

Kumar P., Singh B., Thakur V., Thakur A., Thakur N., Pandey D. and Chand D. (2019). Hyper-production of taxol from Aspergillus fumigatus, an endophytic fungus isolated from Taxus $\mathrm{sp}$. of the Northern Himalayan region, Biotechnology Reports, 24, e00395.

Kumaran R.S., Choi Y.-K., Lee S., Jeon H.J., Jung H. and Kim H.J. (2012). Isolation of taxol, an anticancer drug produced by the endophytic fungus, Phoma betae. African Journal of. Biotechnology, 11, 950-960.

Kumirska J., Czerwicka M., Kaczyński Z., Bychowska A., Brzozowski K., Thöming J. and Stepnowski P. (2010). Application of spectroscopic methods for structural analysis of chitin and chitosan. Marine Drugs, 8, 1567-1636.

Liu K., Ding X., Deng B. and Chen W. (2009). Isolation and characterization of endophytic taxol-producing fungi from Taxus chinensis, Journal of Industrial Microbiology, 36, 1171.

Maharachchikumbura S.S., Hyde K.D., Groenewald J.Z., Xu J. and Crous P.W. (2014). Pestalotiopsis revisited, Studies in Mycology, 79, 121-186.

Markeb A.A., El-Maali N.A., Sayed D.M., Osama A., Abdel-Malek M.A., Zaki A.H., Elwanis M.E. and Driscoll J.J. (2016). Synthesis, structural characterization, and preclinical efficacy of a novel paclitaxel-loaded alginate nanoparticle for breast cancer treatment, International Journal of Breast Cancer, 2016. 
McGahren W., Perkinson G., Growich J., Leese R. and Ellestad G. (1984). Chitosan by fermentation, Process Biochemistry, 19, 88-90.

Mirjalili M.H., Farzaneh M., Bonfill M., Rezadoost H. and Ghassempour A. (2012). Isolation and characterization of Stemphylium sedicola SBU-16 as a new endophytic taxolproducing fungus from Taxus baccata grown in Iran, FEMS Microbiology Letters, 328, 122-129.

Miyoshi H., Shimura K., Watanabe K. and Onodera K. (1992). Characterization of some fungal chitosans, Bioscience, Biotechnology, and Biochemistry, 56, 1901-1905.

Morin-Crini N., Lichtfouse E., Torri G. and Crini G. (2019). Applications of chitosan in food, pharmaceuticals, medicine, cosmetics, agriculture, textiles, pulp and paper, biotechnology, and environmental chemistry, Environmental Chemistry Letters, 17(4), 1667-1692.

Negrea P., Caunii A., Sarac I. and Butnariu M. (2015). The study of infrared spectrum of chitin and chitosan extract as potential sources of biomass, Digest Journal of Nanomaterials and Biostructures (DJNB), 10(4), 1129-1138.

No H.K., Meyers S.P. and Lee K.S. (1989). Isolation and characterization of chitin from crawfish shell waste, Journal of Agricultural and Food Chemistry, 37, 575-579.

Pandi M., Kumaran R.S., Choi Y.-K., Kim H.J. and Muthumary J. (2011). Isolation and detection of taxol, an anticancer drug produced from Lasiodiplodia theobromae, an endophytic fungus of the medicinal plant Morinda citrifolia, African Journal of Biotechnology, 10, 1428-1435.

Park P.-J., Je J.-Y. and Kim S.-K. (2004). Free radical scavenging activities of differently deacetylated chitosans using an ESR spectrometer, Carbohydrate Polymers, 55, 17-22.

Pavia D.L., Lampman G.M., Kriz G.S. and Vyvyan J.A. (2014). Introduction to spectroscopy, 5 th ed. Cengage Learning.

Perdue R.E. (1969). Search for plant sources of anticancer drugs, Morris Arboretum Bull.

Raj K.G., Manikandan R., Arulvasu C. and Pandi M. (2015). Antiproliferative effect of fungal taxol extracted from Cladosporium oxysporum against human pathogenic bacteria and human colon cancer cell line HCT 15, Spectrochimica Acta Part A: Molecular and Biomolecular Spectroscopy, 138, 667674.

Rajendran L., Rajagopal K., Subbarayan K., Ulagappan K., Sampath A. and Karthik G. (2013). Efficiency of fungal taxol on human liver carcinoma cell lines, American Journal of Research Communication, 1, 112-121.

Schmitt P.D., Trasi N.S., Taylor L.S. and Simpson G.J. (2015). Finding the needle in the haystack: characterization of trace crystallinity in a commercial formulation of paclitaxel proteinbound particles by Raman spectroscopy enabled by second harmonic generation microscopy, Molecular Pharmaceutics, 12, 2378-2383.

Shahidi F., Arachchi J.K.V. and Jeon Y.-J. (1999). Food applications of chitin and chitosans, Trends in Food Science \& Technology, 10, 37-51.

Smith N.R. and Dawson V.T. (1944). The bacteriostatic action of rose bengal in media used for plate counts of soil fungi, Soil Science, 58, 467-472.

Stierle A., Strobel G. and Stierle D. (1993). Taxol and taxane production by Taxomyces andreanae, an endophytic fungus of Pacific yew, Science-New York Then Washington, 260, 214214.
Strobel G., Yang X., Sears J., Kramer R., Sidhu R.S. and Hess W. (1996a) ,Taxol from Pestalotiopsis microspora, an endophytic fungus of Taxus wallachiana. Microbiology, 142, 435-440.

Strobel G.A., Hess W., Li J.-Y., Ford E., Sears J., Sidhu R.S. and Summerell B. (1997). Pestalotiopsis guepinii, a taxolproducing endophyte of the wollemi pine, Wollemia nobilis, Australian Journal of Botany, 45, 1073-1082.

Strobel G.A., Hess W.M., Ford E., Sidhu R. and Yang X. (1996b). Taxol from fungal endophytes and the issue of biodiversity, Journal of Industrial Microbiology and Biotechnology, 17, 417-423.

Suffness M. (1995). Taxol: science and applications, CRC press.

Synowiecki J. and Al-Khateeb N.A.A.Q. (1997). Mycelia of Mucor rouxii as a source of chitin and chitosan, Food Chemistry, 60, 605-610.

Tajdini F., Amini M.A., Nafissi-Varcheh N. and Faramarzi M.A. (2010). Production, physiochemical and antimicrobial properties of fungal chitosan from Rhizomucor miehei and Mucor racemosus, International Journal of Biological Macromolecules, 47, 180-183.

Tayel A.A., Gharieb M.M., Zaki H.R. and Elguindy N.M. (2016). Bioclarification of water from heavy metals and microbial effluence using fungal chitosan, International Journal of Biological Macromolecules, 83, 277-281.

Teng W.L., Khor E., Tan T.K., Lim L.Y. and Tan S.C. (2001). Concurrent production of chitin from shrimp shells and fungi, Carbohydrate Research, 332, 305-316.

Vasundhara M., Kuma A. and Reddy M.S. (2016). Molecular approaches to screen bioactive compounds from endophytic fungi, Frontiers in Microbiology, 7.

Vennila R. and Muthumary J. (2011). Taxol from Pestalotiopsis pauciseta VM1, an endophytic fungus of Tabebuia pentaphylla, Biomedicine and Pharmacotherapy, 1, 103-108.

Vennila R., Thirunavukkarasu S. and Muthumarya J. (2010). In vivo studies on anticancer activity of taxol isolated from an endophytic fungus Pestalotiopsis pauciseta Sacc. VM1, Asian Journal of Pharmaceutical and Clinical Research, 3, 30-34.

Wang J., Li G., Lu H., Zheng Z., Huang Y. and Su W. (2000). Taxol from Tubercularia sp. strain TF5, an endophytic fungus of Taxus mairei, FEMS Microbiology Letters, 193, 249-253.

Wani M.C., Taylor H.L., Wall M.E., Coggon P. and McPhail A.T. (1971). Plant antitumor agents. VI. Isolation and structure of taxol, a novel antileukemic and antitumor agent from Taxus brevifolia, Jouran of American Chemical Soceity, 93, 23252327.

Weaver B.A. (2014). How Taxol/paclitaxel kills cancer cells, Molecular Biology of the Cell, 25, 2677-2681.

White S.A., Farina P.R. and Fulton I. (1979). Production and isolation of chitosan from Mucor rouxii, Applied and Environmental Microbiology, 38, 323-328.

Wu T., Zivanovic S., Draughon F.A., Conway W.S. and Sams C.E. (2005). Physicochemical properties and bioactivity of fungal chitin and chitosan, Journal of Agricultural and Food Chemistry, 53, 3888-3894.

Yang Y., Zhao H., Barrero R.A., Zhang B., Sun G., Wilson I.W., Xie F., Walker K.D., Parks J.W. and Bruce R. (2014). Genome sequencing and analysis of the paclitaxel-producing endophytic fungus Penicillium aurantiogriseum NRRL 62431, BMC Genomics, 15, 69. 
Young D., Michelotti E., Swindell C. and Krauss N. (1992). Antifungal properties of taxol and various analogues, Cellular and Molecular Life Sciences, 48, 882-885.

Yuan J., Jian-Nan B., Bing Y. and Xu-Dong Z. (2006). Taxolproducing fungi: a new approach to industrial production of taxol, Chinese Journal of Biotechnology, 22, 1-6.

Zhu L. and Chen L. (2019). Progress in research on paclitaxel and tumor immunotherapy. Cellular \& Molecular Biology Letters, 24(1), 1-11.

Zimoch-Korzycka A., Gardrat C., Al Kharboutly M., Castellan A., Pianet I., Jarmoluk A. and Coma V. (2016). Chemical characterization, antioxidant and anti-listerial activity of nonanimal chitosan-glucan complexes, Food Hydrocolloids, 61, 338-343. 